\title{
Discrete Multi Atlas Segmentation using Agreement Constraints
}

\section{Stavros Alchatzidis ${ }^{134}$}

stavros.alchatzidis@ecp.fr

Aristeidis Sotiras ${ }^{2}$

aristeidis.sotiras@uphs.upenn.edu

Nikos Paragios ${ }^{134}$

nikos.paragios@ecp.fr
${ }^{1}$ Equipe GALEN, INRIA Saclay, Île-de-France, Orsay, France

${ }^{2}$ Section of Biomedical Image Analysis,Department of Radiology, University of Pennsylvania, Pennsylvania, USA

${ }^{3}$ Ecole des Ponts Paristech, Champs-sur-Marne, Île-de-France, France

${ }^{4}$ Ecole Centrale de Paris, Châtenay-Malabry, Île-de-France, France
Atlas-based segmentation describes a class of methods based on the registration of an annotated representative volume to a target one. When a single atlas is used a segmentation of the target image is obtained by warping the annotations using the deformation field found by the registration process. Recently, it has been shown that performing multiple such registrations allows for much improved results comparing to the single atlas case. In Multi Atlas segmentation the target image annotations are produced by fusing the multiple hypotheses either in a local $[2,4]$ or a global [1] fashion. In most methods, the segmentation problem is solved in two discrete steps and registration is merely seen as a fixed preprocessing step.

In this paper, we aim to couple the registration and segmentation problem through a unified formulation for multi-atlas segmentation. Registration terms seek optimal visual correspondences between atlases and target volumes while imposing smoothness. Segmentation terms seek voxel-wise consensus on the labeling of the target with respect to the deformed segmentation maps. Prior per voxel probabilities, produced by learning of local features, are taken into account in a seamless manner. In order to mathematically formulate these components, we adopt a pairwise Markov Random Field (MRF) graphical model where each atlas is associated with a deformation field, while the target image is associated with a segmentation map.

MRF Energy The dicrete pairwise energy function takes the form of:

$$
E_{M R F}(\mathbf{l})=\sum_{p \in \mathcal{V}} g_{p}\left(l_{p}\right)+\sum_{(p, q) \in \mathcal{E}} f_{p q}\left(l_{p}, l_{q}\right)
$$

Graph Structure. Graph $\mathcal{G}$ is made of a set of $N$ isomorphic grid graphs $\mathcal{G}_{D}=\left\{\mathcal{G}_{D_{0}}, \ldots, \mathcal{G}_{D_{N-1}}\right\}$ that represent deformation fields and a set of nodes $\mathcal{V}_{S}$ that represent segmentation. Nodes $p \in \mathcal{V}_{D_{i}}$ encode control points. The solution space around a control point is quantized and indexed by a discrete set of variables $\mathcal{L}_{D}$. This set represents possible control point displacements. We refer to a potential control point displacement attributed to a deformation node by $l^{d}$. The edge system of each grid in $\mathcal{G}_{D}, \mathcal{E}_{D_{i}}$ is created by a regular connectivity scheme.Each node $p \in \mathcal{V}_{S}$ corresponds to a random variable. The set of possible solutions for nodes of $\mathcal{V}_{S}, \mathcal{L}_{S}$ represents the set of anatomical regions augmented by the background label.

The energy in Eq.1 can be seen as broken into 4 terms:

Matching. The matching term, quantifies how well an atlas matches the target image.

$$
g_{p_{i}}^{M}\left(l_{p_{i}}^{d}\right)=\int_{\Omega} \hat{\omega}_{p_{i}}(x) \rho\left(A_{i} \circ D_{i}^{l_{p_{i}}^{d}}, I(x)\right) d x
$$

$D_{i}^{l_{p_{i}}^{d}}$ is the transformation induced by the movement of the control point $p$ in the $i$ th deformation grid by the displacement $l_{p_{i}}^{d}$. The weighting function $\hat{\omega}_{p_{i}}$ determines the contribution of the point $x$ to the unary potential of the control point $p$.

Deformation smoothness. [3] shows that deformation regularization can be efficiently modeled by pairwise potentials by:

$$
f_{p_{i} q_{i}}^{R}\left(l_{p_{i}}^{d}, l_{q_{i}}^{d}\right)=\left\|\mathbf{d}^{l_{p_{i}}^{d}}-\mathbf{d}^{l_{q_{i}}^{d}}\right\|
$$

where $\mathbf{d}^{l_{p_{i}}^{d}}$ is the displacement applied to node $p$ in the $i$-th deformation grid, indexed by $l_{p i}^{d}$.

Segmentation. This term takes into account a per-voxel probability distribution $\pi_{x}(l)$ and is incorporated in the MRF model by setting the unary potentials of the segmentation grid for every label to the negative logprobability of the respective class:

$$
g_{q_{S}}^{S P}\left(l_{q_{S}}^{S}\right)=\int_{\Omega} \hat{\omega}_{q_{S}}(x)\left(-\log \left(\pi_{x}\left(l_{q_{S}}^{S}\right)\right)\right) .
$$

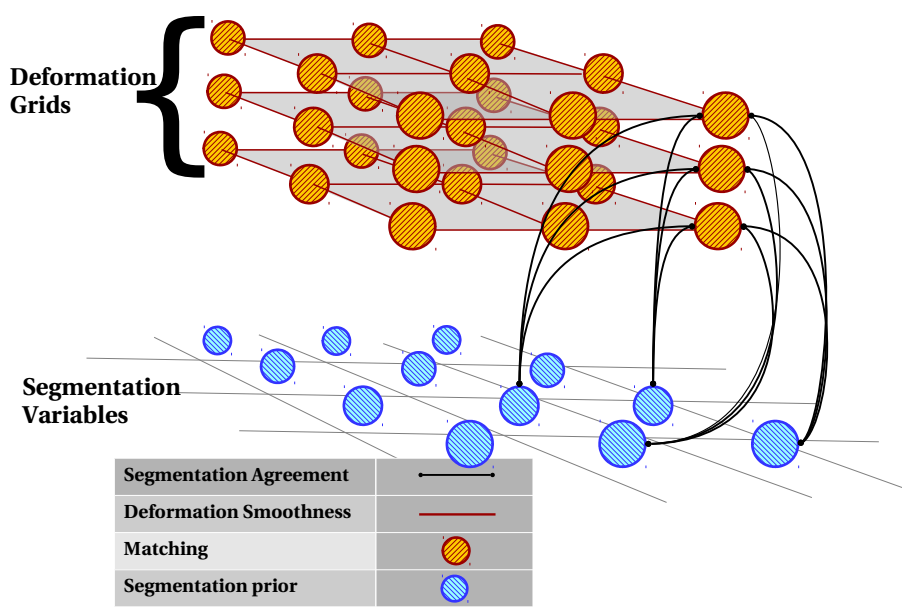

Figure 1: Graph structure of the proposed pairwise model.

where $\hat{\omega}_{q S}(x)$ denotes the support of the segmentation node. For our experiments we have corresponded each segmentation node to a voxel, but a coarser or softer assignment could be envisaged.

Integrated Segmentation and Multi-Atlas Registration. To encourage the agreement between the estimated segmentation and the warped segmentation we penalize control point displacements of grid $\mathcal{G}_{D_{i}}$ that result in the warped segmentation mask corresponding to atlas $i$ not agreeing with our final segmentation:

$f_{p_{i} q_{S}}^{C}\left(l_{p_{i}}^{d}, l_{q_{S}}^{s}\right)=\int_{\Omega} \hat{\omega}_{q_{S}}(x) \hat{\omega}_{p_{i}}(x) \hat{\rho}\left(A_{i} \circ D_{i}^{l_{p_{i}}^{d}}, I(x)\right) \operatorname{Ind}\left(S_{i} \circ D_{i}^{l_{p_{i}}^{d}}(x), l_{q_{S}}^{s}\right) d x$

where $p_{i}$ belongs to the grid $\mathcal{G}_{D_{i}}$ and $q_{S}$ belongs to $\mathcal{G}_{S} . \operatorname{Ind}(x, y)=1$ except from $\operatorname{Ind}(x, x)=0$.

Validation. Comparing to independent pairwise registrations, our method is shown to increase registration quality in terms of overlap and harmonic energy. In addition, concensus between hypotheses is enforced leading to more concordant pairwise registrations, rejecting agressive deformation fields produced merely by good matchings.In addition, classical label fusion methods are outperformed by the annotations produced by our method.

[1] P. Aljabar, R.A. Heckemann, A. Hammers, J.V. Hajnal, and D. Rueckert. Multi-atlas based segmentation of brain images: Atlas selection and its effect on accuracy. NeuroImage, 46(3):726-738, 2009.

[2] X. Artaechevarria, A. Munoz-Barrutia, and C. Ortiz-de Solorzano. Combination Strategies in Multi-Atlas Image Segmentation: Application to Brain MR Data. Medical Imaging, IEEE Transactions on, 28(8):1266-1277, August 2009.

[3] Ben Glocker, Aristeidis Sotiras, Nikos Komodakis, and Nikos Paragios. Deformable Medical Image Registration: Setting the State of the Art with Discrete Methods*. Annual Review of Biomedical Engineering, 13(1):219-244, 2011.

[4] I. Isgum, M. Staring, A. Rutten, M. Prokop, M. A. Viergever, and B. van Ginneken. Multi-Atlas-Based Segmentation With Local Decision Fusion. Application to Cardiac and Aortic Segmentation in CT Scans. Medical Imaging, IEEE Transactions on, 28(7):1000-1010, July 2009 . 